Feasibility Study

\title{
Computer-Tomographic Verification of Ultrasound-Guided Piriformis Muscle Injection: A Feasibility Study
}

Gustavo Fabregat, MD1, Marta Roselló, MD', Juan M. Asensio-Samper, MD', Vicente L. Villanueva-Pérez, MD, PhD², Vicente Martínez-Sanjuan, MD, PhD ${ }^{3}$, José De Andrés, $\mathrm{MD}, \mathrm{PhD}^{4}$, and Urs Eichenberger, $\mathrm{MD}, \mathrm{PhD}^{5}$

\begin{abstract}
From: ${ }^{1}$ Anesthesiologist, Multidisciplinary Pain Management Department, General University Hospital, Valencia, Spain; ${ }^{2}$ General Practitioner, Multidisciplinary Pain Management Department, General University Hospital, Valencia, Spain; ${ }^{3}$ Head of Radiology Department, General University Hospital, Valencia, Spain; ${ }^{4}$ Professor of Anesthesia, School of Medicine of Valencia; Chief of Anesthesia, Critical Care and Multidisciplinary Pain Management Department, General University Hospital,

Valencia, Spain; ${ }^{5}$ Head of

Department of Anesthesia, Intensive Care and Pain Medicine, St. Anna Clinic, Lucerne, Switzerland.
\end{abstract}

Address Correspondence: Gustavo Fabregat, MD NAvda. Tres Cruces s/n, 46014, General University Hospital, Valencia, Spain

E-mail: gfabregat@gmail.com

Disclaimer: There was no external funding in the preparation of this manuscript. Conflict of interest: Each author certifies that he or she, or a member of his or her immediate family, has no commercial association (i.e., consultancies, stock ownership, equity interest, patent/licensing arrangements, etc.) that might pose a conflict of interest in connection with the submitted manuscript.

Manuscript received: 02-24-2014 Revised manuscript received: 07-24-2014 Accepted for publication: 07-29-2014

Free full manuscript: www.painphysicianjournal.com
Background: Approximately $6 \%$ to $8 \%$ of lumbar pain cases, whether associated with radicular pain or not, may be attributed to the presence of piriformis muscle syndrome. Available treatments, among others, include pharmacotherapy, physical therapy, and injections of different substances into the muscle. Various methods have been used to confirm correct needle placement during these procedures, including electromyography (EMG), fluoroscopy, computed tomography (CT), or magnetic resonance imaging (MRI). Ultrasonography (US) has now become a widely used technique and therefore may be an attractive alternative for needle guidance when injecting this muscle.

Objective: The objective of this study was to assess the reliability of US in piriformis injection of patients with piriformis syndrome.

Study Design: Feasibility study; 10 patients with piriformis muscle syndrome were injected with botulinum toxin A using a US-guided procedure. Then patients were administered $2 \mathrm{~mL}$ iodinated contrast and were then transferred to the CT scanner, where they underwent pelvic and hip imaging to assess intramuscular distribution of the iodinated contrast.

Setting: Multidisciplinary Pain Management Department in Spain.

Results: Of all 10 study patients ( 8 women, 2 men), 9 had intramuscular or intrafascial contrast distribution. Distribution did not go deeper than the piriformis muscle in any of the patients. The absence of contrast (intravascular injection) was not observed in any case.

Limitations: The main limitation of our study is the use of ionizing radiation as confirmation technique.

Conclusion: Ultrasound-guided puncture may be a reliable and simple procedure for injection of the piriformis muscle, as long as good education and training are provided to the operator. US has a number of advantages over traditional approaches, including accessibility and especially no ionizing radiation exposure for both health care providers and patients.

Key words: Piriformis, ultrasound-guidance, $C T$, pain, chronic pain, ultrasonography, botulinum toxin, sciatica

Pain Physician 2014; 17:507-513 
T he piriformis muscle is a deep muscle that originates from the anterior part of the sacrum with digitations attached to the bone between the anterior sacral foramina. It exits the pelvis through the greater sciatic foramen. It is pyramidal in shape and inserts into the upper border and medial side of the greater trochanter of the femur by a round tendon (1). The piriformis muscle is closely related to important vascular and nervous structures which also exit the pelvis through the greater sciatic notch, such as the superior and inferior gluteal vessels and nerves, and the sciatic nerve (2). The sciatic nerve leaves the pelvic cavity just beneath the lower border of the piriformis muscle and above the sacrotuberous ligament, through the greater sciatic notch. This is the most common anatomical configuration, but there are multiple variations; for instance, in some individuals, the sciatic nerve passes above the piriformis muscle; in others, a divided sciatic nerve passes above and below the muscle, or even below and through (3). The primary action of the piriformis muscle is the lateral rotation of the hip, along with the gemellus inferior and superior, obturator externus and internus, and quadratus femoris (4).

The symptoms of the "piriformis syndrome" may be very varied. There may be pain and even paresthesia in the lower back and groin regions, perineum, buttocks, sacroiliac region, greater trochanter and posterior thigh regions. Piriformis syndrome has been reported to cause sciatica (5). Approximately $6 \%$ to $8 \%$ of lumbar pain cases, whether associated with radicular pain or not, may be attributed to the presence of piriformis syndrome (6). It is difficult to diagnose piriformis syndrome, as there are no uniform criteria or specific diagnostic tests; therefore, in many cases, diagnosis is established by exclusion (6). Available treatments include physical therapy, stretching and strengthening exercises, and the injection of different substances such as local anesthetics and botulinum toxin into the muscle $(5,7,8)$. Different methods have been used to confirm the correct placement of the needle when injection therapies are administered, including electromyography (EMG) (9), fluoroscopy (10), computed tomography (CT) (7), or magnetic resonance imaging (MRI) (11). Among the available imaging techniques, there have been substantial advances in ultrasonography (US) applications for interventional pain management procedures. Unlike CT or fluoroscopy, US techniques provide continuous real-time identification of soft tissue, vessels, and nerves without exposing the patient or the operator to radiation. In addition, technological development has produced smaller, compact US machines, offering highquality images and portability, which confer US optimal availability and usability (12). A number of studies have been conducted to validate US-guided piriformis injection techniques, including cadaveric studies (13), a comparison to fluoroscopy (12), EMG confirmation (14), and more recently MRI verification (15).

The primary objective of our study was to validate the precision of US-guided piriformis injection by conducting a CT scan immediately after the US procedure, which included the administration of a radiopaque contrast medium. In addition, anthropometric data were collected from the study patients and skin-to-piriformis distance and fatty layer thickness were measured on the CT scans.

\section{Methods}

After approval by the Ethics Committee and in order to assess the reliability of US for piriformis muscle injection, we selected 10 consecutive patients with symptoms suggestive of piriformis syndrome to attempt piriformis injection of botulinum toxin. All patients had favorable responses to a previous run-in test with injection of a local anesthetic into the piriformis. The test was performed under fluoroscopic guidance by a physician not involved in the study. Patients gave their written informed consent before being included in the study. All US procedures were performed using a portable Logiq e US device (General Electric Medical Systems, Milwaukee, WI). A 4C-RS convex transducer (General Electric Medical Systems, Milwaukee, WI) was used, as it has a frequency range of $1.8 \mathrm{MHz}$ to $6 \mathrm{MHz}$. Frequency settings were adjusted individually for optimal visualization, based on the patient's body constitution. The harmonic imaging option was activated to reduce artifacts and improve image quality (16). Patients were placed in the prone position and the buttock area was exposed for the US examination. All procedures were performed by the same health care provider (GF), an anesthesiologist specialized in the treatment of pain with over five years experience in the use of US techniques for diagnostic and therapeutic procedures in pain management and regional anesthesia.

The greater trochanter was identified by palpation on the side to be treated. A line was drawn from the greater trochanter to the midpoint of the lateral border of the sacrum. This superficial line runs approximately parallel to the piriformis and was used to determine the angle of the transducer for optimal alignment with the muscle (Fig. 1A). An initial examination was 


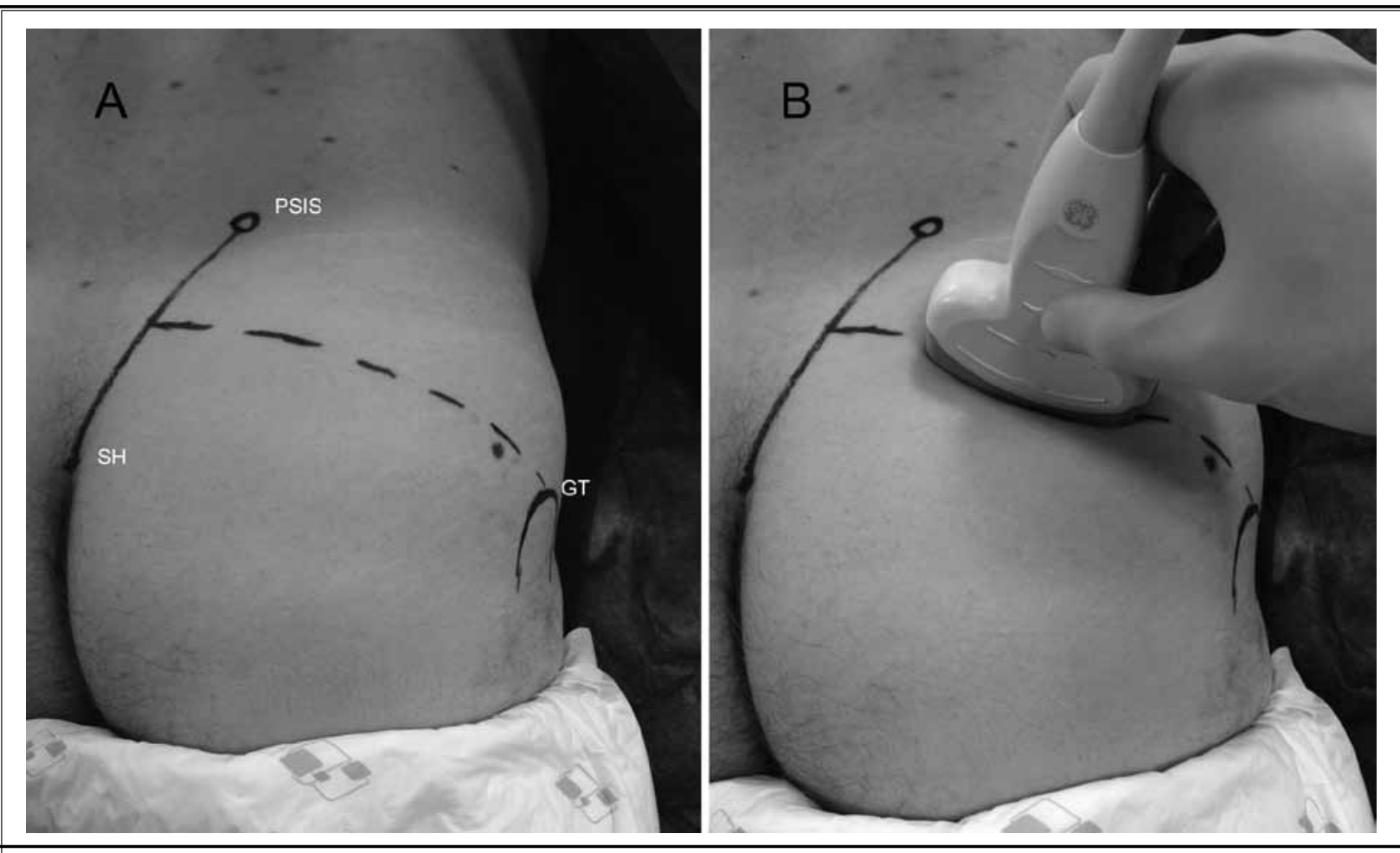

Fig. 1. (A) Surface landmarks. The line between the posterior superior iliac spine (PSIS) and the sacral hiatus (SH) defines approximately the lateral border of the sacrum. The superficial line connecting the midpoint of the sacral border and the upper border of the greater trochanter (GT) runs approximately parallel to the piriformis muscle.

(B) Image showing the orientation of the transducer to achieve the alignment of the ultrasound plane to the course of the piriformis muscle.

performed to locate the approximate area in which the piriformis muscle lies. The region to be treated was then disinfected with topical $2 \%$ chlorhexidine and sterile dressings were placed to confine the region. The treated area was then examined with the US transducer using a sterile probe cover (Sterile Kit, Bard Access Systems, Salt Lake City, UT) and a sterile US transmission gel (Aquasonic 100, Parker Laboratories, Inc., Fairfield, $\mathrm{NJ})$. Following the technique described by Smith's group (12), the posterior superior iliac spine (PSIS) was scanned by initially palpating it and the transducer was then placed perpendicularly to the longitudinal axis of the body. While maintaining this position, the transducer was moved caudally until the hyperechoic signal of the ilium was lost, indicating that the ultrasound plane moves into the greater sciatic notch. With the medial end of the transducer remaining at the lateral end of the sacrum, the piriformis muscle appears deep to the lateral sacral border. In contrast, the gluteus maximus muscle runs superficial to the sacrum (Fig. 2A). Because the piriformis muscle runs laterally and slightly caudally toward its insertion at the superior greater trochanter and above the posterior part of the acetabulum, the position of the transducer was corrected by rotating it and aligning the ultrasound plane to the course of the muscle, taking the line drawn on the surface as a guide (Fig. 1B) (14). Correct visualization of the piriformis was confirmed by asking patients to flex the ipsilateral knee by $90^{\circ}$ and externally rotating the hip joint to see the piriformis muscle move under the gluteus maximus.

After the piriformis muscle was correctly identified, a $90 \mathrm{~mm}$ 22G Quincke spinal needle (Vygon, Ecouen, France) was advanced laterally along the long axis of the transducer toward the piriformis, until the tip was placed in the belly of the muscle. In cases where the ultrasound image showed a deeper target, a $150 \mathrm{~mm}$ $21 \mathrm{G}$ neurostimulation needle was used (Locoplex®, Vygon, Ecouen, France). A syringe was prefilled with 100 IU botulinum toxin A (Botox, Allergan, Irvine, CA) reconstituted in normal saline to a total volume of $5 \mathrm{~mL}$. This solution was administered under US visualization into the muscle. Without changing the position 


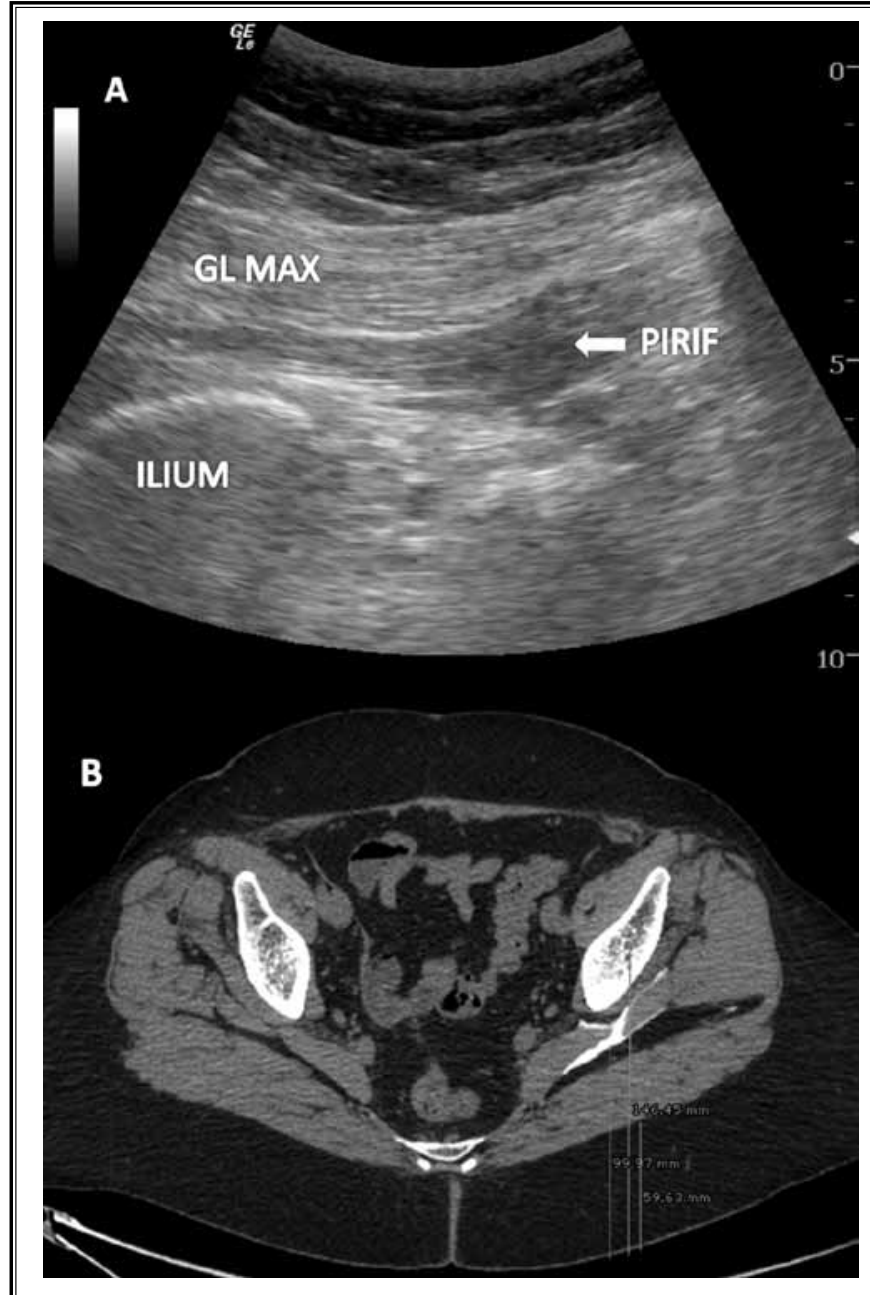

Fig. 2. (A) Ultrasonographic image of the piriformis muscle in the longitudinal plane obtained with a convex transducer at 1-6 $\mathrm{MHz}$. The piriformis can be seen deeper to the gluteus maximus and passing laterally toward the greater trochanter (GT). (B) Computed tomography scan. CT slice at approximately the sacrococcygeal symphysis. In the patient's left side, distribution of the contrast is clearly seen in the piriformis muscle, deep under the gluteus maximus. Measurements are shown from the border of the skin to the midpoint of the ipsilateral ilium: distance from the skin to the posterior border of the piriformis (Distsk-pir, white arrow), and distance from the skin to the posterior border of the gluteus maximus (Distsk-gm, open arrow).

of the needle, we injected $2 \mathrm{~mL}$ iodinated contrast thereafter (Optiray, loversol $350 \mathrm{mg} / \mathrm{mL}$, Mallinckrodt, St. Louis, MO).

Patients were then transferred to the CT scanner, where they underwent pelvic and hip imaging to assess intramuscular distribution of the iodinated contrast. All patients were placed in the supine position for this procedure. Images were taken using a 64-channel CT scanner
(VCT, GEH, Waukesha, WI). An initial scout view was taken with anteroposterior and lateral projections. Based on these projections, a helical scan was taken from approximately L5-S1 down to the lesser trochanter. A dosage of $120 \mathrm{kV}$ and $203 \mathrm{mAs}$ was used with $1.25 \mathrm{~mm}$ slices. The total dose was approximately $34.6 \mathrm{mGy}$.

The images and contrast dye distribution were analyzed by a radiologist and 2 different measurements were obtained for all patients. Successful injection was defined as intramuscular or intrafascial piriformal distribution of the contrast dye. The slice passing through the sacrococcygeal symphysis was identified on the CT sequence. A perpendicular straight line was then drawn on the scan, from the midpoint of the hipbone to the border of the skin. Patient measurements were taken using this line, from the border of the skin to the posterior border of the piriformis muscle (Distsk-pir); a second measurement was then taken from the border of the skin to the posterior border of the gluteus maximus (Distsk-gm), providing an estimate of the amount of subcutaneous fat in the treated region (Fig. 2B).

\section{Results}

Table 1 summarizes the patients' anthropometric data. Median age was 49 years (IQR $37-$ 56 years), median height was $160 \mathrm{~cm}$ (IQR -156$170 \mathrm{~cm}$ ), and median body mass index (BMI) was $29.7 \mathrm{Kg} / \mathrm{m} 2$ (IQR $23.9-32.4 \mathrm{Kg} / \mathrm{m} 2$ ). No adverse events occurred during the US procedure and the procedure was tolerated well by all patients. No anatomical variants in the piriformis muscle region were found in the study patients. Of the 10 patients included in the study ( 8 women and 2 men), 9 showed intramuscular or intrafascial distribution of the iodinated contrast dye, which was defined as success of the US technique. In only one case, the presence of the main portion of contrast was seen in the anatomical plane between the piriformis and the gluteus maximus muscles. lodinated contrast was not seen to be present beyond the piriformis muscle in any of the patients. In addition, no case of absence of contrast was reported, which would have been a sign of an intravascular injection. The median distance from the skin to the posterior border of the piriformis (Distsk-pir) was $79.80 \mathrm{~mm}$ (IQR $57.03-95.05 \mathrm{~mm}$ ) and the median fatty layer 
Table 1. Summary of the study patients' anthropometric data. Success was defined as injected contrast dye within the muscle sheath or in the muscle belly controlled by CT scan.

\begin{tabular}{|c|c|c|c|c|c|c|c|c|}
\hline Id. & Age & Height (cm) & Weight (kg) & BMI & Dist sk-pir & Dist sk-gm & Gender & Success \\
\hline Case 1 & 45 & 161 & 78 & 30.09 & 79.79 & 36.10 & Female & Yes \\
\hline Case 2 & 52 & 175 & 140 & 45.71 & 97.34 & 52.14 & Female & No \\
\hline Case 3 & 29 & 160 & 76 & 29.69 & 79.80 & 29.09 & Female & Yes \\
\hline Case 4 & 36 & 175 & 80 & 26.12 & 53.64 & 24.10 & Male & Yes \\
\hline Case 5 & 60 & 153 & 50 & 21.36 & 56.27 & 36.51 & Female & Yes \\
\hline Case 6 & 55 & 168 & 70 & 24.80 & 72.80 & 26.76 & Male & Yes \\
\hline Case 7 & 45 & 160 & 76 & 29.69 & 85.97 & 52.14 & Female & Yes \\
\hline Case 8 & 53 & 158 & 76 & 30.44 & 94.29 & 53.33 & Female & Yes \\
\hline Case 9 & 60 & 153 & 50 & 21.36 & 57.28 & 38.59 & Female & Yes \\
\hline Case 10 & 37 & 157 & 94 & 38.14 & 99.97 & 59.63 & Female & Yes \\
\hline Median & 48.5 & 160 & 76 & 29.69 & 79.80 & 37.55 & & \\
\hline IQR & $36-57$ & $156-170$ & $65-84$ & $23.9-32.4$ & $57.03-95.05$ & $28.51-52.44$ & & \\
\hline
\end{tabular}

thickness (Distsk-gm) in this area was $37.55 \mathrm{~mm}$ (IQR $28.51-52.44 \mathrm{~mm}$ ). No patients had adverse effects and all of them were discharged within the same day as planned.

\section{Discussion}

As far as we know, this is the first study performed in patients with piriformis muscle syndrome to validate US-guided injection into the piriformis muscle by using an image technique.

In 9 of the 10 treated patients the contrast was found in the piriformis muscle as targeted, in none it was found deep to the muscle and in only one patient - the one with the highest body mass index of 45.7 $\mathrm{kg} / \mathrm{m}^{2}$ and a fatty layer more than $5 \mathrm{~cm}$ thick - it was found in the fascia layer between the piriformis and the gluteus maximus muscles. Difficulty in performing US-guided interventions accurately in obese patients is well known $(17,18)$ and the major limitation of the use of ultrasound. In obese patients, because of the deep anatomic location of the target structures, the US beam travels greater distances, resulting in beam attenuation (16). In addition, other factors may affect imaging quality through fat. The fat tissue, for instance, has a nonlinear relationship to the absorption dependent on the US-frequency used compared to the usually assumed linear relationship in most other biological tissues. This nonlinear relationship of fat results in exaggerated attenuation of the US beam in adipose individuals. In addition, when the US beam travels through a muscle-fat interface, part of the energy reflects back to the transducer because of the mismatch of acoustic impedance between the tissues (19). Therefore, there are more reflective interfaces in obese patients, not only leading to more echoes but also decreasing incident sound available to penetrate deeper tissues (16). All these factors can contribute to suboptimal image quality and lead to a suboptimal needle placement and injection. Blindly or fluoroscopically controlled injections may end up with an even higher inaccuracy in obese patients and have other major limitations as explained below.

The use of US-based techniques for interventional procedures in patients with chronic pain has grown significantly over the last few years. However, compared to the use of US in regional anesthesia, it is still limited. One obvious explanation could be: in regional anesthesia, US-guided nerve location is compared to "blind" location techniques (such as neurostimulation, external anatomical landmarks, etc.), whereas for the treatment of pain, an imaging technique such as US is compared to other imaging techniques, especially fluoroscopy and $\mathrm{CT}$, which are considered to be the current gold standard in this setting (20).

US has a number of obvious advantages over other imaging techniques used for locating and injecting the piriformis muscle. Similarly to fluoroscopy and CT, US can easily be used to identify bony structures as landmarks for locating target structures but neither patients nor the operators are exposed to ionizing radiation. In addition, US is, like MRI, an imaging technique of 
choice for examining soft tissue (21). Major advantages of ultrasound compared to MRI are the lower cost of the equipment and the real time visualization of the structures. Vessels and nerves are visualized in real time and continuously, as are the fascial planes between the muscles. Even the needle can be seen as it travels along the anatomical planes, to determine the safest trajectory and avoid accidental damage to structures at risk. US can be used for patients who have contraindications for other imaging techniques (e.g., pregnancy, contrast allergies, etc.). Technological development has produced smaller, portable US devices with excellent image performance, which can be used anywhere without having to move patients to specific facilities, as is the case for MRI or CT. In addition, because no specific high price equipment is needed beyond the portable US machine and because the procedures can be performed in the pain unit offices, we presume that USguided procedures have the potential to bring health care costs down.

Several studies have demonstrated the reliability of US for locating and injecting the piriformis muscle. Finnoff et al (13) injected the piriformis muscles in 10 unembalmed cadavers with liquid latex comparing fluoroscopically guided contrast-controlled and USguided injection techniques. Accuracy of injection as assessed at cadaver dissection was significantly better in the US-guided injection group (95\% versus 30\%). Other authors have compared both techniques in cadavers and, more importantly, in patients. The study of Smith et al (12) provides an in-depth description of the USguided technique and demonstrates the validity of the procedure; however, no formal determination of the injection accuracy was undertaken.

In other studies the positioning of the needle for injection of the piriformis was verified using EMG (14) or motor stimulation of the muscle (22). Real patients were used in these studies, but their primary limitation is that they are single case reports and that the confirmatory techniques they used (EMG and motor stimulation) may not be considered to be highly accurate for positioning, as they can be artifacted by the presence of nearby muscles with similar actions as the piriformis. We believe that positioning should be validated using imaging techniques in which the muscle and the injected material are identified without any uncertainty.

A group of German researchers (15) recently validated the use of US for piriformis injection using MRI control. They reported $90 \%$ accuracy of needle placement with the US technique ( 9 out 10 cases), but the study was developed in healthy volunteers. Howerer this rate of success is very similar to our findings and the rates reported by other authors (13).

We believe that techniques should also be validated in real scenarios with real patients, not only in ideal conditions (e.g., using healthy volunteers). It has been hypothesized that muscular hypertrophy, increased tone, or traumatic alterations and anatomical variants of the piriformis muscle may contribute to the symptoms experienced by patients $(3,23,24)$. It remains unclear whether these conditions, with the exception of anatomical variants, could have an impact on the US image of the muscle in patients with piriformis syndrome. The 10 consecutive patients included in our study were responding well to a muscular injection runin test with local anesthetic. Therefore, the chance they were really suffering from a pirifomis muscle pathology was high. However, no anatomical variants or other US-visible pathologies were found in these patients. Another limitation to the use of healthy patients is that they may not be anthropometrically representative of the patients seen in pain management units. No anthropometric data are reported in the study by Blunk et al (15); however, it could be assumed that they had thin fatty layers, as the mean distance measured from the skin to the target area was only $4.6 \mathrm{~cm}$. In our study, the mean distance to the posterior border of the piriformis was $7.7 \mathrm{~cm}$ and some patients had fatty layers thicker than $5 \mathrm{~cm}$.

The main limitation of our study is the use of CT as confirmation technique. Although CT obtains lower quality images of soft tissues when compared to MRI and exposes the patient to ionizing radiation, it is one of the imaging techniques used routinely to perform interventional procedures for the treatment of pain $(7,25,26)$, it allows a good visualization of soft tissue, and it is cheaper than MRI. In addition, using a small amount of contrast dye (similar to the one used to perform the procedure under fluoroscopy) allows us to see clearly the distribution of the injected among the soft tissue.

\section{Conclusion}

In conclusion, US-guidance for piriformis muscle injection is a promising method to perform such interventions. The present study was able to confirm the accuracy of US-guided injection of the piriformis muscle by CT imaging validation in all examined patients with piriformis muscle syndrome with the exception of one morbidly obese patient. This and previous studies 
demonstrate that US-guidance for piriformis injection is reliable and reproducible, as long as the operator is adequately trained. The use of US has the potential to revolutionize the field of interventional pain management, as it already did in the field of regional anesthesia. However, published data about US-guided treatments are still very limited. Further studies should focus on outcome and safety of US-guided pain interventions compared to traditional imaging techniques such as fluoroscopy.

\section{References}

1. Netter F. Atlas de Anatomía Humana. 4th ed. Editorial Masson, Madrid, 2002.

2. Rohen JW, Yokochi C, Lütjen-Drecoll E. Atlas de Anatomía Humana. Estudio Fotográfico del Cuerpo Humano. 5th ed. Elsevier España S.A., Madrid, 2003.

3. Benzon HT, Katz JA, Benzon HA, Iqbal MS. Piriformis syndrome: Anatomic considerations, a new injection technique, and a review of the literature. Anesthesiology 2003; 98:1442-1448.

4. Moore KL. Anatomía Humana con Orientación Clínica. Lippincott Williams and Wilkins, Madrid, 2010.

5. Fishman LM, Dombi GW, Michaelsen C, Ringel S, Rozbruch J, Rosner B, Weber C. Piriformis syndrome: Diagnosis, treatment, and outcome - a 10-year study. Arch Phys Med Rehabil 2002; 83:295-301.

6. Halin R. Sciatic pain and the piriformis muscle. Postgrad Med 1983; 74:69-72.

7. Fanucci E, Masala S, Sodani G, Varrucciu V, Romagnoli A, Squillaci E, Simonetti G. CT-guided injections of botulinum toxin for percutaneous therapy of piriformis muscle syndrome. Eur Radiol 2001; 11:2543-2548.

8. Jeynes LC, Gauci CA. Evidence for the use of botulinum toxin in the chronic pain setting - a review of the literature. Pain Pract 2008; 8:269-276.

9. Fishman $\mathrm{SH}$, Caneris OA, Bandman TB, Audette JF, Borsook D. Injection of the piriformis cuscle by fluoroscopic and electromiographic guidance. Regional Anesth Pain Med 1998; 23:554-559.

10. Betts A. Combined fluoroscopic and nerve stimulator technique for injection of the piriformis muscle. Pain Physician 2004; 7:279-281.

11. Filler AG, Haynes J, Jordan SE, Prager J,
Villablanca JP, Farahani K, McBride DQ, Tsuruda JS, Morisoli B, Batzdorf U, Johnson JP. Sciatica of nondisc origin and piriformis syndrome: diagnosis by magnetic resonance neurography and interventional magnetic resonance imaging with outcome study of resulting treatment. J Neurosurg Spine 2005; 2:99-115.

12. Smith J, Hurdle MF, Locketz AJ, Wisniewski SJ. Ultrasound-guided piriformis injection: Technique description and verification. Arch Phys Rehabil 2006; 87:1664-1667.

13. Finnoff JT, Hurdle MF, Smith J. Accuracy of ultrasound-guided versus fluoroscopically guided contrast-controlled piriformis injections: A cadaveric study. J Ultrasound Med 2008; 27:1157-1163.

14. Chen H, Takemoto R, Hata J. Ultrasound guided piriformis injection with confirmation of needle placement through electromyography. Pain Med 2012; 13: 978-979.

15. Blunk JA, Nowotny M, Scharf J, Benrath J. MRI Verification of ultrasound-guided infiltrations of local anesthetics into the piriformis muscle. Pain Med 2013; [Epub ahead of print].

16. Saranteas T. Limitations in ultrasound imaging techniques in anesthesia: Obesity and muscle atrophy? Anesth Analg 2009; 109:993-994.

17. Marhofer P, Pilz-Lubsczyk B, Lönnqvist PA, Fleischmann E. Ultrasound-guided peripheral regional anaesthesia: A feasibility study in obese versus normalweight women. Int J Obes (Lond) 2013; [Epub ahead of print].

18. Rauch S, Kasuya Y, Turan A, Neamtu A, Vinayakan A, Sessler DI. Ultrasoundguided lumbar medial branch block in obese patients: A fluoroscopically con- firmed clinical feasibility study. Reg Anesth Pain Med 2009; 34:340-342.

19. Shmulewitz A, Teefey SA, Robinson BS. Factors affecting image quality and diagnostic efficacy in abdominal sonography: A prospective study of 140 patients. J Clin Ultrasound 1993; 21: 623-630.

20. Narouze S. Ultrasonography in pain medicine: A sneak peak at the future. Pain Practice 2008; 8:223-225.

21. Brown AK, O'Connor PJ, Roberts TE, Wakefield RJ, Karim Z, Emery P. Recommendations for musculoskeletal ultrasonography by rheumatologists: Setting global standards for best practice by expert consensus. Arthritis Rheum 2005; 53:83-92.

22. Huerto AP, Yeo SN, Ho KY. Piriformis muscle injection using ultrasonography and motor stimulation--report of a technique. Pain Physician 2007; 10:687-690.

23. Benson ER, Schutzer SF. Posttraumatic piriformis syndrome: Diagnosis and results of operative treatment. J Bone Joint Surg Am 1999; 81:941-949.

24. Chen WS. Bipartite piriformis muscle: An unusual cause of sciatic nerve entrapment. Pain 1994; 58:269-272.

25. Masala S, Crusco S, Meschini A, Taglieri A, Calabria E, Simonetti G. Piriformis syndrome: Long-term follow-up in patients treated with percutaneous injection of anesthetic and corticosteroid under CT guidance. Cardiovasc Intervent Radiol 2012; 35:375-382.

26. Fanucci E, Masala S, Sodani G, Varrucciu V, Romagnoli A, Squillaci E, Simonetti G. CT-guided injection of botulinic toxin for percutaneous therapy of piriformis muscle syndrome with preliminary MRI results about denervative process. Eur Radiol 2001; 11:2543-2548. 
